\title{
Ferritin: A potential serum marker for lymph node metastasis in head and neck squamous cell carcinoma
}

\author{
ZHANGWEI HU $^{1 *}$, LEI WANG ${ }^{1 *}$, YONG HAN ${ }^{2}$, FEN LI $^{3}$, ANYUAN ZHENG $^{1}$, YONG XU ${ }^{1}$, \\ FEI WANG ${ }^{1}$, BOKUI XIAO ${ }^{1,3}$, CHEN CHEN $^{3}$ and ZEZHANG TAO ${ }^{1,3}$ \\ ${ }^{1}$ Department of Otolaryngology Head and Neck Surgery, Renmin Hospital of Wuhan University, Wuhan, Hubei 430060; \\ ${ }^{2}$ Clinical Research Institute, Zhejiang Provincial People's Hospital, Hangzhou, Zhejiang 310014; \\ ${ }^{3}$ Research Institute of Otolaryngology Head and Neck Surgery, Renmin Hospital of \\ Wuhan University, Wuhan, Hubei 430060, P.R. China
}

Received February 17, 2018; Accepted September 20, 2018

DOI: $10.3892 / \mathrm{ol} .2018 .9642$

\begin{abstract}
Head and neck squamous cell carcinoma (HNSCC) is the sixth most common cancer in the world, yet current treatment options are associated with limited success. The aim of the present study was to investigate the expression of ferritin in HNSCC and clarify whether it may serve as a biomarker for predicting HNSCC metastasis. The chemiluminescent immunoassay method was used to investigate the differences in the serum ferritin (SF) levels between patients with and without tumors, and between HNSCC with and without lymph node metastasis. The iron content and expression levels of ferritin were detected to verify the differences between tumor and normal tissues, and between HNSCC without and with lymph node metastasis. Data from the Gene Expression Omnibus (GEO) dataset was used to support the aforementioned results. No statistically significant difference in the SF level was observed between patients with and without tumors. Iron content and expression levels of ferritin heavy chain (FTH) and ferritin light chain (FTL) were higher in tumor tissues compared with normal tissues. The iron content and expression levels of SF, FTH and FTL were increased in HNSCC with metastasis compared with HNSCC without metastasis. The GEO dataset further verified the results and reported that the expression level of FTH was correlated with the prognosis of patients with HNSCC. Ferritin may not be a biomarker for
\end{abstract}

Correspondence to: Dr Zezhang Tao, Department of Otolaryngology Head and Neck Surgery, Renmin Hospital of Wuhan University, 99 Zhangzhidong Street, Wuhan, Hubei 430060, P.R. China E-mail: taozezhang@hotmail.com

Dr Chen Chen, Research Institute of Otolaryngology Head and Neck Surgery, Renmin Hospital of Wuhan University, 99 Zhangzhidong Street, Wuhan, Hubei 430060, P.R. China

E-mail: chenchen_md@whu.edu.cn

"Contributed equally

Key words: head and neck squamous cell carcinoma, ferritin, metastasis, Gene Expression Omnibus dataset, biomarker the early diagnosis of HNSCC. However, an association exists between the expression level of ferritin and HNSCC cervical metastasis. SF may be a potential biomarker for predicting cervical lymph node metastasis in patients with HNSCC.

\section{Introduction}

Head and neck squamous cell carcinoma (HNSCC) is the sixth most common cancer in the world (1). A total of $\sim 600,000$ new cases are reported every year, with a marked proportion of these cases in China (2). HNSCC frequently occurs as a heterogeneous tumor with an aggressive phenotype (3). In clinical practice, metastasis, particularly cervical lymph node metastasis, frequently occurs during the progression of the disease (4). Regional cervical lymph node metastasis is closely associated with the poor prognosis of patients with HNSCC (5). Current treatments for HNSCC have limited success in improving patient prognosis. Therefore, identifying potential novel markers may be a promising strategy to treat HNSCC.

Iron metabolism is associated with tumor growth and promotes tumor cell proliferation in $\operatorname{HNSCC}(6,7)$. The major storage form of iron in the human body is ferritin (8). Ferritin has been studied for nearly 80 years. However, it remains a key molecule, with novel characteristics continuing to be discovered. Recent studies revealed that the expression levels of ferritin were closely associated with a number of malignant tumors, including lung cancer (9) and primary hepatocellular carcinoma (10). However, the role of ferritin in HNSCC tumorigenesis and development is not fully understood.

In the present study, serological and histological experiments, in addition to Gene Expression Omnibus (GEO) datasets, were used to investigate the expression levels of ferritin in HNSCC. The primary aim was to clarify whether ferritin may serve as a biomarker for HNSCC diagnosis and metastasis prediction.

\section{Materials and methods}

Patient selection for serumferritin (SF) detection. As presented in Table I, 281 patients, including 44 patients with mucosal 
inflammation (nasopharyngeal epithelium or throat mucous membrane), 133 with benign tumors (vocal polyps, $n=108$; cyst of epiglottis, $n=25$ ), 20 with precancerosis (vocal leukoplakia, $\mathrm{n}=1$; atypical hyperplasia, $\mathrm{n}=19$ ) and 84 with cancer (carcinoma in situ, $\mathrm{n}=14$; HNSCC without metastasis, $\mathrm{n}=40$; HNSCC with metastasis, $n=30$ ), were selected for the study. The inclusion criteria were: i) patient diagnoses were based on conventional clinical, radiographic and histopathological or cytological criteria; ii) patients had previously undergone primary tumor resection. The 70 patients with HNSCC (not including carcinoma in situ) additionally underwent appropriate cervical lymph node dissection at Renmin Hospital of Wuhan University (Wuhan, China) between January 2013 and January 2015. The exclusion criteria were: i) patients with severe systemic disorders (such as diabetes mellitus, renal or heart failure); ii) patients with diseases that affect SF levels (such as liver injury, precancerous anemia or other types of cancer); iii) patients who underwent previous chemotherapy or radiotherapy.

Serum sample collection and analyses. Venous blood samples (3-5 ml) were collected in a plain vial at diagnosis, prior to surgery. The samples were stored at room temperature for $2 \mathrm{~h}$. Serum samples were obtained following centrifugation at $1,500 \times \mathrm{g}$ at $4^{\circ} \mathrm{C}$ for $5 \mathrm{~min}$ and immediately stored at $-70^{\circ} \mathrm{C}$ until use. The expression levels of SF were detected using the chemiluminescent immunoassay method with a Siemens Centaur XP fully-automated chemiluminescence immunoassay analyzer (Siemens Healthcare GmbH, Erlangen, Germany) in the Department of Clinical Laboratory, Renmin Hospital of Wuhan University, and the correlative Quantitative Assay kit for ferritin (cat. no. 012245) was purchased from Siemens Healthcare GmbH.

Ethics statement. The present study was approved by the ethics committee of Renmin Hospital of Wuhan University. All specimens were collected from patients who provided written informed consent, in accordance with the principles of the Declaration of Helsinki.

Patient selection for iron staining and immunohistochemical staining. A total of 70 sets of paraformaldehyde-fixed paraffin-embedded specimens were obtained from the pathology department, including 40 patients without and 30 with cervical node metastasis. Details are presented in Table II. Each set contained one primary tumor tissue and one corresponding cervical lymph node tissue. The tissues were fixed in $4 \%$ formaldehyde for $24 \mathrm{~h}$ at $4^{\circ} \mathrm{C}$, and were routinely processed into paraffin blocks. Each tissue was divided into at least three paraffin-embedded sections: One for detecting iron content using iron staining; and the remaining two for detecting ferritin $\mathrm{H}(\mathrm{FTH})$ and ferritin L (FTL), respectively, using immunohistochemistry (IHC).

Neck color Doppler ultrasonography. The neck lymph nodes of the 70 patients with HNSCC were detected using color Doppler ultrasonography prior to surgery, and the results were obtained from the radiology department. The features of the nodes included size (longitudinal diameter, LD) and shape (long axis/short axis or L/S ratio). For size, the dividing line was $1 \mathrm{~cm} \mathrm{LD}$, and for the shape, $2 \mathrm{x} \mathrm{L/S}$ ratio.

Tissue microarray for IHC. Two tissue microarrays (TMAs) (no. HN803b) with the same patients' information were purchased from US Biomax, Inc. (Rockville, MD, USA) to examine differences in the expression of FTH and FTL between HNSCC and normal tissues. Each TMA consisted of 11 normal and 69 HNSCC tissue samples, with the mean age of patients being 53.4 years (range 15-90 years). Details of the TMA characteristics are presented in Table III.

Iron staining. Iron staining (also termed Prussian blue staining) was performed using a $5 \%$ potassium hexacyanoferrate trihydrate and hydrochloric acid solution. Staining incubation time was $\sim 30 \mathrm{~min}$ at room temperature. Subsequently, the sections were rinsed with water, counterstained with nuclear fast red, dehydrated, and covered.

IHC staining. The 3-5 $\mu \mathrm{m}$ sections were deparaffinized with standard pure xylene for $15 \mathrm{~min}$, three times at room temperature and hydrated in an alcohol gradient. PBS was used to wash the sections. Antigen retrieval was performed in boiling citrate buffer ( $\mathrm{pH} 6.0$ ) for $15 \mathrm{~min}$. The sections were cooled down to room temperature in the citrate buffer. Following washing of the sections with PBS three times for $5 \mathrm{~min}, 0.3 \%$ hydrogen peroxide phosphate-citrate buffer was used to block endogenous peroxidase activity for $10 \mathrm{~min}$ at room temperature. Goat serum (5\%; Fuzhou Maixin Biotech Co., Ltd., Fuzhou, China) was used to block the samples for $10 \mathrm{~min}$ at room temperature. The sections were then rinsed with PBS for 5 min and incubated with primary antibody against FTL (cat. no. SAB2108636; 1:500 dilution; Sigma-Aldrich, Merck KGaA, Darmstadt, Germany) and against FTH (cat. no. SAB2108662; 1:250 dilution; Sigma-Aldrich, Merck $\mathrm{KGaA}$ ) for $12 \mathrm{~h}$ at $4^{\circ} \mathrm{C}$. The sections were incubated with the biotinylated goat anti-rabbit secondary antibody (cat. no. SP KIT-C9; 1:250 dilution; Fuzhou Maixin Biotech Co., Ltd.) for $30 \mathrm{~min}$ at room temperature. The slides were stained with diaminobenzidine for $5 \mathrm{~min}$ at room temperature. Hematoxylin was used to counterstain the nucleus for $5 \mathrm{~min}$ at room temperature, followed by dehydration, and mounting.

Evaluation of iron staining and IHC. Images of the stained sections were captured using an Olympus BX40 light microscope and CC-12 Soft-Imaging System (Olympus Corporation, Tokyo, Japan). The sections were analyzed and scored for intensity (0-3) and frequency (0-4). The intensity was scored as follows: Grade (0) negative; grade (1) weak intensity; grade (2) moderate intensity; and grade (3) strong intensity. The frequency scores were assigned when 0-25, 26-50, 51-75 and $76-100 \%$ of the tumor cells were positive, respectively. For statistical analysis, the intensity and frequency were transformed into a Composite Expression Score (CES) using the formula: CES=intensity $x$ frequency. The range of CES was $0-12$. The CES was scored as negative (0) weak positive (1-4) positive (5-8) or strong positive (9-12).

GEO dataset. The GSE33205 (Cancer Outlier Gene Profile Sets Elucidate Pathways and patient-Specific Targets in Head 
Table I. Baseline characteristics of the 281 patients.

\begin{tabular}{|c|c|c|c|c|c|c|}
\hline \multirow[b]{2}{*}{ Group } & \multicolumn{2}{|c|}{ Sex } & \multicolumn{2}{|c|}{ Age, years } & \multicolumn{2}{|c|}{ Menopausal status } \\
\hline & Male & Female & Range & Mean & Pre-menopausal & Post-menopausal \\
\hline Inflammation & 23 & 21 & $13-72$ & 45 & 13 & 8 \\
\hline Benign & 48 & 85 & $5-73$ & 46 & 53 & 32 \\
\hline Precancerosis & 16 & 4 & $29-73$ & 53 & 4 & 0 \\
\hline Cancer & 82 & 2 & $31-84$ & 61 & 0 & 2 \\
\hline
\end{tabular}

Table II. Characteristics of patients selected.

\begin{tabular}{|c|c|c|c|}
\hline Characteristic & Metastasis group $(n=30)$ & No metastasis group $(n=40)$ & P-value \\
\hline Sex & & & $>0.05$ \\
\hline Male & 30 & 39 & \\
\hline Female & 0 & 1 & \\
\hline Age at diagnosis & & & $<0.05$ \\
\hline Median (range) & $59.7(31-73)$ & $61.2(41-81)$ & \\
\hline Tumor type & & & $<0.05$ \\
\hline Glottis & 15 & 37 & \\
\hline Hypopharynx & 10 & 2 & \\
\hline Supraglottis & 2 & 0 & \\
\hline Nasopharynx & 3 & 0 & \\
\hline Tonsil & 0 & 1 & \\
\hline Differentiation grade & & & $>0.05$ \\
\hline Well & 25 & 32 & \\
\hline Moderate & 5 & 7 & \\
\hline Poor & 0 & 0 & \\
\hline Total & 30 & 40 & $>0.05$ \\
\hline
\end{tabular}

and Neck Squamous Cell Carcinoma), GSE6631 (Expression Data from Head and Neck Squamous Cell Carcinoma) and GSE27020 (Identification and Validation of a Multi-gene Predictor of Recurrence in Primary Laryngeal Cancer) datasets were obtained from the GEO (https://www.ncbi.nlm. nih.gov/). The expression values of FTH and FTL were transformed into relative expression values, and expression levels were compared between HNSCC and normal tissues, using GEO2R software (https://www.ncbi.nlm.nih.gov/geo/geo2r/).

Statistical analysis. All statistical analyses were performed using SPSS version 20.0 (IBM Corporation, Armonk, NY, USA). Quantitative data are expressed as the mean \pm standard deviation. Each test was performed at least three times. A Student's t-test was used to compare the differences between two groups. One-way analysis of variance (ANOVA) was performed for the comparison of multiple groups. If the results of the ANOVA indicated significant differences, post-hoc analysis was performed with the Tukey test. The diagnostic accuracy was evaluated using receiver operating characteristic (ROC) curve analysis. A 4 -fold Table $\chi^{2}$ test was used to determine the sensitivity and specificity of size and shape. Correlation analysis was performed using Pearson's correlation
Table III. Tissue microarray characteristics.

\begin{tabular}{lcc}
\hline & \multicolumn{2}{c}{ Group } \\
\cline { 2 - 3 } Total $n=80$ & HNSCC $(\mathrm{n}=69)$ & Normal $(\mathrm{n}=11)$ \\
\hline Sex & 57 & 5 \\
Male & 12 & 6 \\
Female & & 28.8 \\
Age, years & 57.3 & $15-48$ \\
Mean & $32-90$ & \\
Range & &
\end{tabular}

coefficient. $\mathrm{P}<0.05$ was considered to indicate a statistically significant difference.

\section{Results}

SF levels are higher in patients with HNSCC and metastasis compared with patients with HNSCC without metastasis. All patients were divided into four groups: i) Inflammation; 

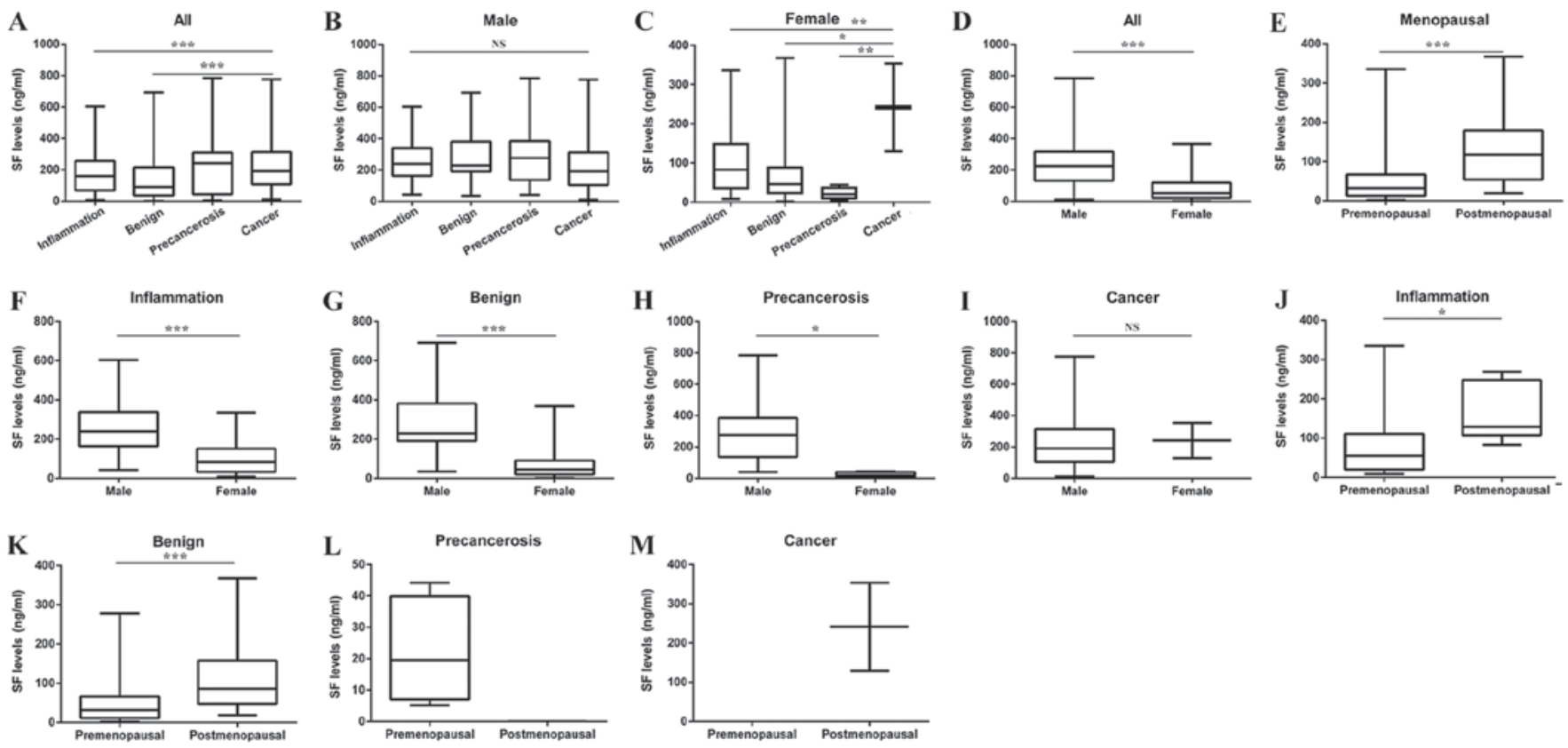

Figure 1. Overall analysis of the SF level in different groups. (A) SF level in the inflammation, benign tumor, precancerosis and cancer groups (F=6.614). (B) Difference in the SF level between different groups of male patients ( $F=0.739)$. (C) Difference in the SF level between different groups of female patients ( $F=5.209)$. The $\mathrm{SF}$ level was significantly higher in the cancer group compared with the benign, precancerossis and inflammation groups. (D) Difference in the SF level between male and female patients ( $t=12.00)$. (E) Difference in the SF level between pre- and postmenopausal female patients ( $t=-5.098)$. ( $F)$ Difference in the SF level between male and female patients in the inflammation group ( $t=4.036)$. (G) Difference in the SF level between male and female patients in the benign tumor group ( $\mathrm{t}=9.377$ ). (H) Difference in the SF level between male and female patients in the precancerosis group ( $\mathrm{t}=2.716$; $\mathrm{P}=0.014)$. (I) Differences in the $\mathrm{SF}$ level between male and female patients in the cancer group $(\mathrm{t}=-0.018 ; \mathrm{P}=0.985)$. (J) Difference in the $\mathrm{SF}$ level between premenopausal and postmenopausal women in the inflammation group ( $\mathrm{t}=-2.359 ; \mathrm{P}=0.03)$. $(\mathrm{K})$ Difference in the $\mathrm{SF}$ level between premenopausal and postmenopausal women in the benign tumor group ( $\mathrm{t}=-4.147)$. (L) Difference in the SF level between premenopausal and postmenopausal women in the precancerosis group. (M) Difference in the SF level between premenopausal and postmenopausal women in the cancer group. ${ }^{*} \mathrm{P}<0.05,{ }^{* *} \mathrm{P}<0.01,{ }^{* * * *} \mathrm{P}<0.001$. NS, not significant; SF, serum ferritin.

ii) benign tumor; iii) precancerosis; and iv) cancer. The SF level in the cancer group was higher compared with the benign group (Fig. 1A). However, in male patients, no significant difference was reported among the four groups (Fig. 1B). In female patients, the SF level in the cancer group was significantly higher compared with the benign and precancerosis groups (Fig. 1C). The differences in the SF levels between male and female patients were compared, and the SF level of male patients was significantly higher compared with that of female patients (Fig. 1D). Postmenopausal women had a higher SF level compared with premenopausal women (Fig. 1E).

The differences in the SF level between male and female patients were compared in each group. In general, male patients had a higher SF level compared with female patients (Fig. 1F-I). Taking menopausal status into consideration, the SF level was reported to be higher in postmenopausal women compared with premenopausal women in the benign and inflammation groups (Fig. 1J-M).

The SF level was compared between carcinoma in situ $(n=14)$ and HNSCC without metastasis groups $(n=40)$. No significant difference was observed between these two groups (Fig. 2A). Therefore, carcinoma in situ was considered as a part of the HNSCC without metastasis group. Subsequently, the cancer group was divided into two subgroups according to the cervical metastasis status: HNSCC with metastasis, $n=30$; HNSCC without metastasis, $n=54$. It was revealed that the SF level in the HNSCC with metastasis group was significantly higher compared with the HNSCC without metastasis group (Fig. 2B). The ROC analysis revealed that the area under the curve (AUC) for SF to predict cervical metastasis was 0.842 , and the cutoff value of the SF level was $205.55 \mathrm{ng} / \mathrm{ml}$ (Fig. 2C).

Doppler ultrasonography may be less optimal for metastasis prediction compared with SF. The Doppler results of the 70 patients in the cancer group were collected to further examine the importance of SF in metastasis prediction. No statistically significant difference in SF was reported between male and female patients in the cancer group (Fig. 1I). The results of the Doppler and SF levels are presented in Table IV. No statistically significant difference in L/S ratio (Fig. 2D) and LD (Fig. 2E) was noted between these two groups, yet the SF level (Fig. 2F) exhibited a statistically significant difference. The sensitivity of LD and the L/S ratio for detecting metastasis was 60 and 20\%, and the specificity was 37.5 and 10\%, respectively. The ROC analysis of the SF level revealed that the AUC for metastasis was 0.862 , the cutoff value of the SF level was $205.60 \mathrm{ng} / \mathrm{ml}$ (Fig. 2G), and the sensitivity and specificity of SF for predicting neck metastasis were 86.7 and $80.0 \%$, respectively.

Ferritin expression levels are higher in the tumor tissues of HNSCC with metastasis. Tissue samples of the cancer group were obtained from the pathology department to clarify whether the expression of ferritin was also upregulated in the tumor tissues of HNSCC with metastasis. IHC detection demonstrated that whether in primary tumor or lymph node 
Table IV. Doppler and SF results in 70 HNSCC patients.

\begin{tabular}{|c|c|c|c|c|c|c|}
\hline \multirow[b]{2}{*}{ Variable } & \multicolumn{2}{|c|}{$\mathrm{LD}(\mathrm{cm})$} & \multicolumn{2}{|c|}{$\mathrm{L} / \mathrm{S}$ ratio } & \multicolumn{2}{|c|}{$\mathrm{SF}(\mathrm{ng} / \mathrm{ml})$} \\
\hline & $<1$ & $\geq 1$ & $<2$ & $\geq 2$ & $<205.60$ & $\geq 205.60$ \\
\hline Metastasis & 12 & 18 & 6 & 24 & 4 & 26 \\
\hline No metastasis & 15 & 25 & 4 & 36 & 31 & 9 \\
\hline P-value & $>0.05$ & & $>0.05$ & & $<0.001$ & \\
\hline
\end{tabular}

LD, Longitudinal diameter; L/S, long axis/short axis; SF, serum ferritin; HNSCC, head and neck squamous cell carcinoma.

A

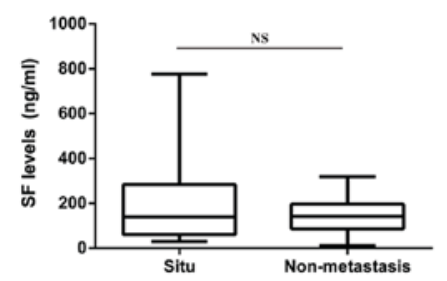

B

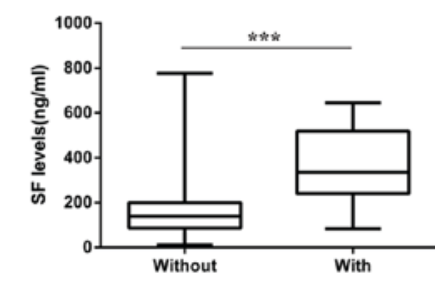

F

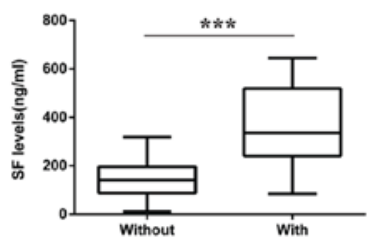

C



G



Figure 2. Differences in the SF level between the carcinoma in situ and non-metastasis groups, and between the HNSCC groups with and without metastasis. (A) Carcinoma in situ vs. HNSCC without metastasis ( $\mathrm{t}=0.759 ; \mathrm{P}=0.457)$. (B) HNSCC with vs. without cervical lymph node metastasis ( $\mathrm{t}=-5.928)$. (C) ROC curve of the SF level for predicting cervical lymph node metastasis in patients with HNSCC (AUC $=0.842 ; \mathrm{P}<0.001$; Youden's index, max $=0.636$; sensitivity $=86.7 \%$; specificity $=76.9 \%$; and $\mathrm{SF}=205.55 \mathrm{ng} / \mathrm{ml}$ ). (D) Difference in the $\mathrm{L} / \mathrm{S}$ ratio between the HNSCC with and without metastasis groups. (E) Difference in longitudinal diameter between the HNSCC without and with metastasis groups. (F) Difference in the SF level between the HNSCC without and with metastasis groups. (G) ROC curve of the SF level for predicting cervical lymph node metastasis in patients with HNSCC (cancer in situ not included) $($ AUC $=0.862$; $\mathrm{P}<0.001$; Youden's index, $\max =0.667$; sensitivity $=86.7 \%$; specificity $=80.0 \%$; and cutoff value of $\mathrm{SF}=05.60 \mathrm{ng} / \mathrm{ml})$. ${ }^{* * *} \mathrm{P}<0.001$. AUC, area under the curve; $\mathrm{NS}$, not significant; ROC, receiver operating characteristic; SF, serum ferritin; L/S, long axis/short axis; HNSCC, head and neck squamous cell carcinoma.

tissues, levels of FTH (Fig. 3A) and FTL (Fig. 3B) were higher in the HNSCC with metastasis group compared with the HNSCC without metastasis group. Iron staining (Fig. 3C) demonstrated that the iron content was higher in the HNSCC with metastasis group compared with the HNSCC without metastasis group, in primary tumor and lymph node tissues. Furthermore, the correlation analysis illustrated that the iron content was significantly correlated with SF, FTH and FTL (Fig. 3D).

Expression levels of FTH and FTL are upregulated in HNSCC tissues. Based on the above findings, two TMAs were purchased to investigate the differences in the protein expression levels of FTH and FTL between HNSCC and normal tissues. However, unlike the expression of ferritin in serum, the protein expression levels of FTH (Fig. 4A and B) and FTL (Fig. 4C and D) were significantly higher in HNSCC tissues compared with normal tissues.
The associated data from the GEO dataset were downloaded and analyzed. Using GSE33205 and GSE6631, the relative expression levels of FTH (Fig. 5A and B) and FTL (Fig. 5C and D) were compared between HNSCC and normal tissues. The gene expression levels of FTH and FTL were higher in HNSCC compared with normal tissues, which was consistent with the histochemical findings. Furthermore, the results of GSE27020 revealed that a high expression level of FTH was associated with poor survival in patients with HNSCC (Fig. 5E). However, no data relating to the association between FTL and survival were reported.

\section{Discussion}

Ferritin has a typical structure of a 24-subunit spherical protein encapsulating an iron oxide core (11). The subunit comprises two different types: FTH $(21 \mathrm{kDa})$ and FTL (19 kDa) (12). Only FTH has the enzymatic activity to 
A
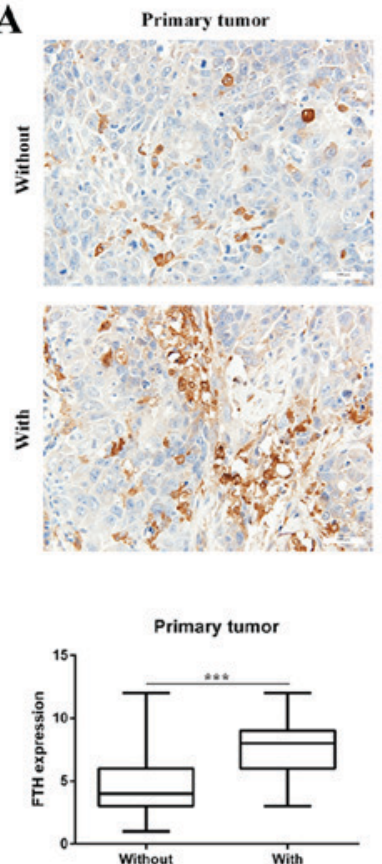

C
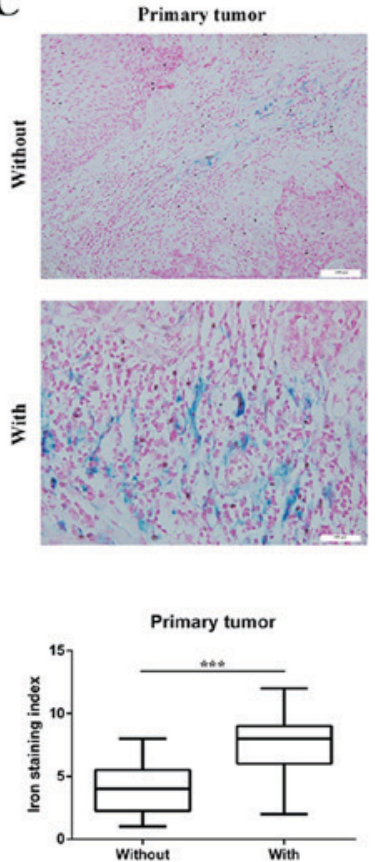
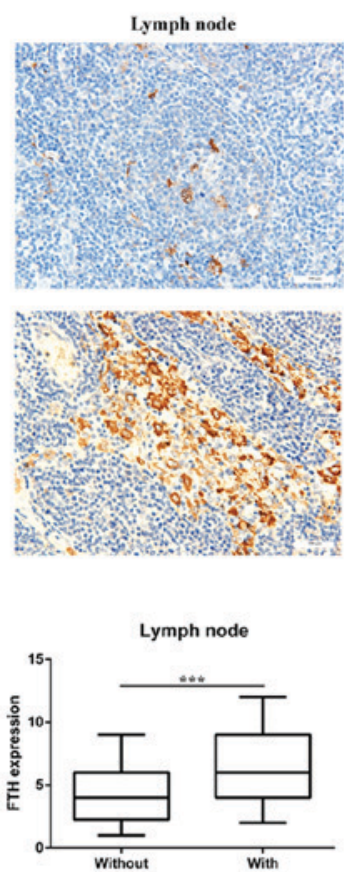

Lymph node
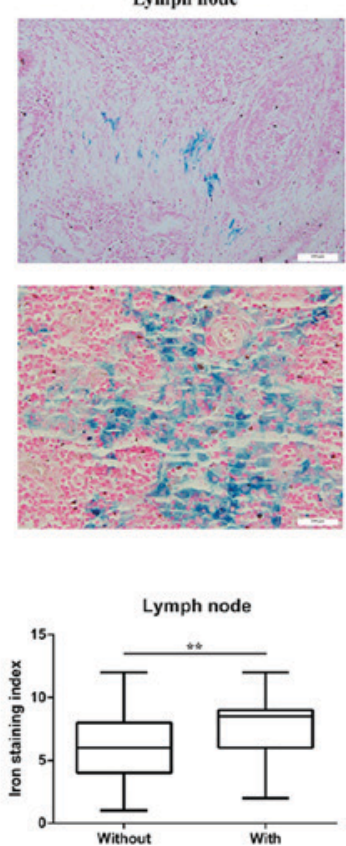
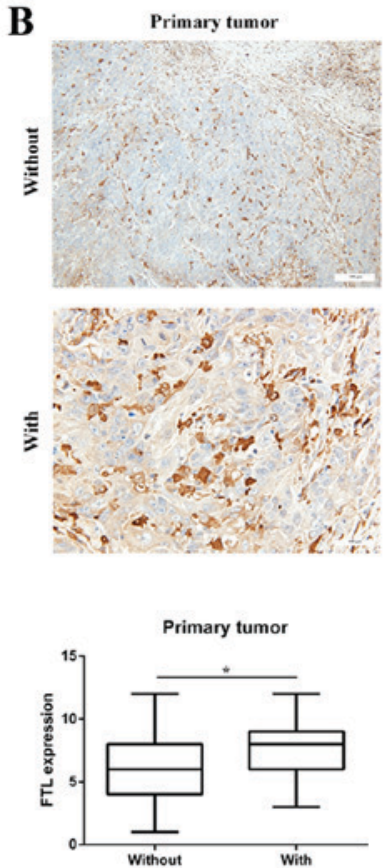

D
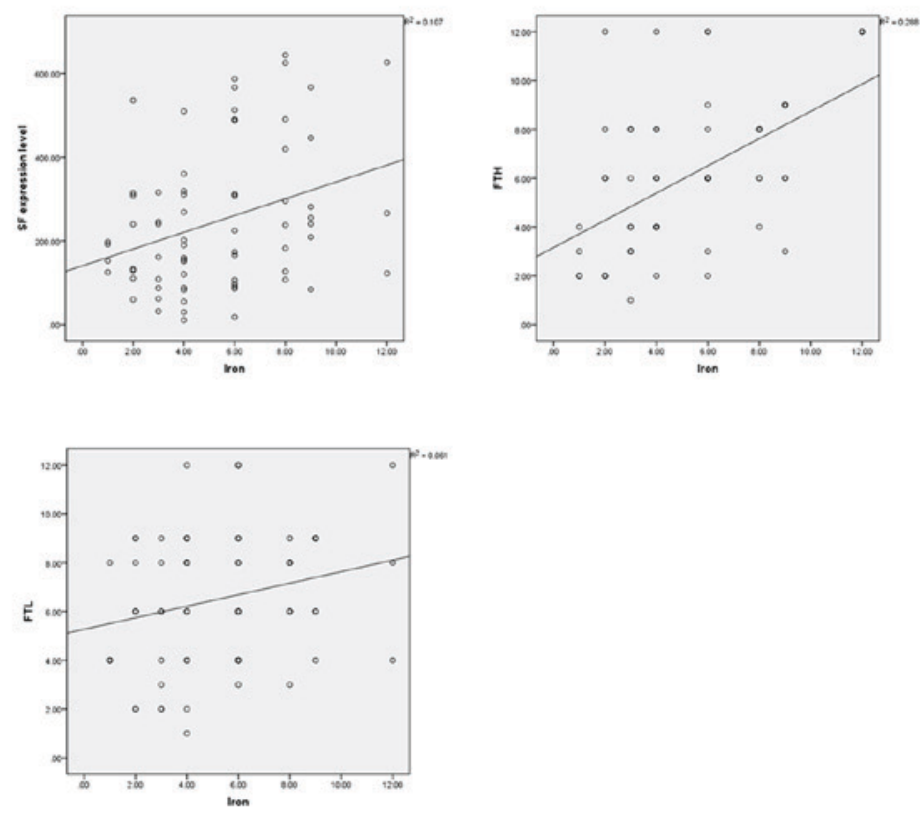
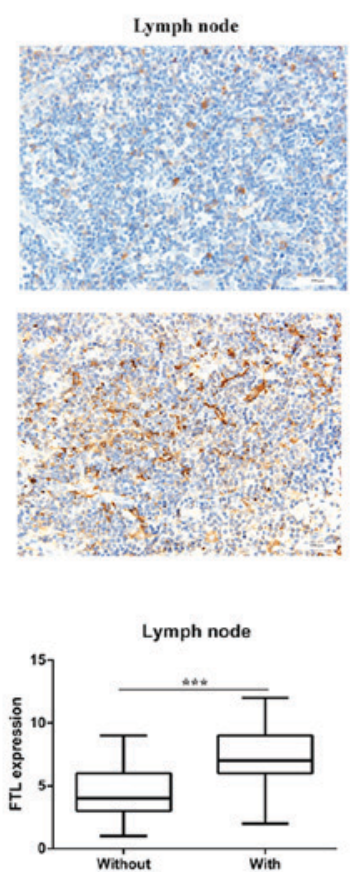

Figure 3. Ferritin expression levels are higher in HNSCC tumor tissues with metastasis. (A) Expression levels of FTH in 70 patients with HNSCC. Whether in primary tumor $(\mathrm{t}=3.948)$ or lymph node tissue $(\mathrm{t}=3.511)$, the expression of FTH was higher in the HNSCC with metastasis group compared with the HNSCC without metastasis group. Magnification, x200. (B) Expression levels of FTL in 70 patients with HNSCC. Whether in primary tumor (t=2.609) or lymph node tissue ( $\mathrm{t}=3.710)$, the expression levels of FTL were higher in the HNSCC with metastasis group compared with the HNSCC without metastasis group. Magnification, $x 200$. (C) Iron content in 70 patients with HNSCC. Whether in primary tumor ( $\mathrm{t}=6.262)$ or lymph node tissue ( $\mathrm{t}=3.192)$, the iron content was higher in the HNSCC with metastasis group compared with the HNSCC without metastasis group. Magnification, x200. (D) Correlation between iron content and FTH levels, and the protein expression level of FTL in 70 patients with HNSCC. Iron content was significantly correlated with $\mathrm{SF}$ levels ( $\mathrm{r}=0.327$; $\mathrm{P}<0.01$ ), FTH levels $(r=0.537 ; \mathrm{P}<0.001)$ and FTL levels $(\mathrm{r}=0.247 ; \mathrm{P}<0.05)$. ${ }^{*} \mathrm{P}<0.05,{ }^{* *} \mathrm{P}<0.01,{ }^{* * *} \mathrm{P}<0.001$. NS, not significant; FTH, ferritin H subunit; FTL, ferritin L subunit; SF, serum ferritin; HNSCC, head and neck squamous cell carcinoma.

convert $\mathrm{Fe}^{2+}$ into $\mathrm{Fe}^{3+}(13,14)$. The $\mathrm{SF}$ level was higher in male patients compared with female patients. Additionally, the SF level was higher in postmenopausal women compared with premenopausal women (15). These results are in accordance with previous findings. This may be due to menstruation, which causes a loss of $\sim 250 \mathrm{mg}$ iron per year (16). Undiminished iron may lead to a higher SF level in postmenopausal women.

Currently, the correlation between iron metabolism and tumor development has become a major concern $(17,18)$. Numerous studies have revealed that the expression levels of ferritin are upregulated in various types of tumors, including 
A

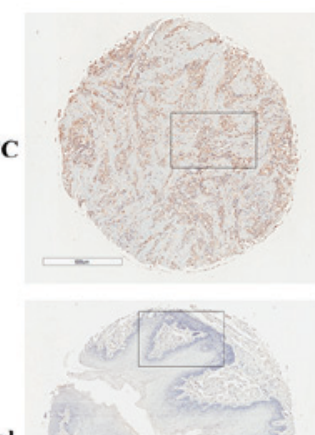

Normal

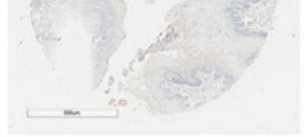

C



HNSCC

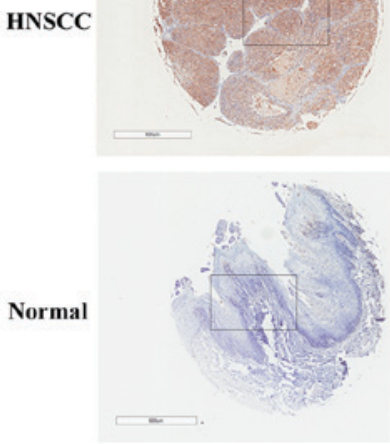

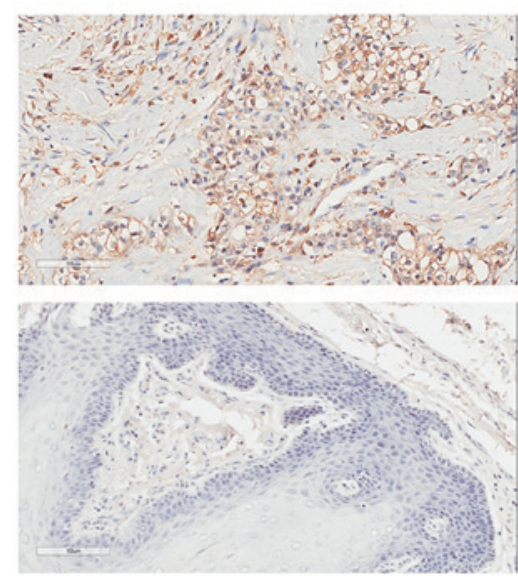


B

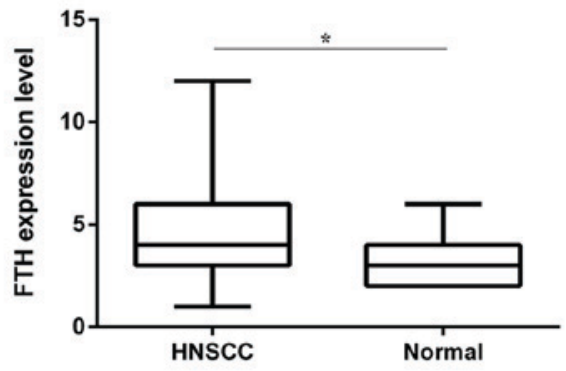

D

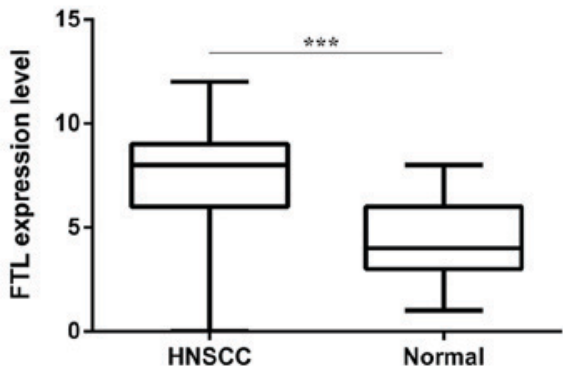

Figure 4. FTL and FTH levels in HNSCC and normal tissue. (A) IHC analysis of the expression levels of FTH in HNSCC and normal tissues. Magnification: left panel, x38; right panel, x200. (B) Differences in FTH expression levels between HNSCC and normal tissues ( $\mathrm{t}=2.161)$. (C) IHC analyzing the expression levels of FTL in HNSCC and normal tissues. Magnification: left panel, x38; right panel, x200. (D) Differences in FTL expression levels between HNSCC and normal tissue ( $\mathrm{t}=3.521) .{ }^{*} \mathrm{P}<0.05,{ }^{* * *} \mathrm{P}<0.001$. FTH, ferritin H subunit; FTL, ferritin L subunit; HNSCC, head and neck squamous cell carcinoma; IHC, immunohistochemistry.

lung cancer and prostate cancer (19). The present study demonstrated that the iron content and expression levels of ferritin were higher in HNSCC compared with normal tissues. As reported by other studies, iron metabolism is vital for normal cell function (20). However, in tumor growth, endocytosis leads to the storage of a large amount of iron in cancer cells (21).

On the one hand, the expression of transferrin receptor 1 (TFR1) is upregulated in the tumor membrane and transports increased levels of iron into cancer cells $(22,23)$. On the other hand, the major protein that transports iron out of cells is ferroportin, and its expression is always downregulated in cancer cells $(24,25)$. Therefore, a large amount of iron is stored in cancer cells and subsequently promotes the expression of ferritin. With the development of the tumor, high levels of ferritin are secreted or leaked from damaged tumor cells, leading to a higher SF level (26). Notably, no statistically significant difference in the SF level was reported between HNSCC and benign tumors or inflammation in the present study. This may be due to that fact that in a few pathological cases, disturbance in iron metabolism may lead to a higher SF level (27). Therefore, SF may be not a potential serum marker for the early diagnosis of HNSCC.

The present study reported that the expression levels of ferritin were significantly higher in the HNSCC with metastasis group compared with the HNSCC without metastasis group. This is the first time, to the best of our knowledge, that this phenomenon has been described. A study indicated that ferritin may regulate vascular remodeling and angiogenesis. It was also demonstrated that ferritin may block the antiangiogenic effects of cleaved high-molecular-weight kininogen (HKa) through specific binding to the antiangiogenic domain of $\mathrm{HKa}$ (28). This may be a possible mechanism; however, whether it promotes the metastasis of HNSCC to cervical lymph nodes requires investigation.

Data from the GEO dataset demonstrated that high expression levels of FTH were associated with poor survival. A similar phenomenon has recently been described in breast cancer and astrocytic brain tumors $(29,30)$. However, no relevant data about the prognostic value of FTL in HNSCC are available. Growing evidence suggests that artificial iron compounds may inhibit tumor growth and cell proliferation (31-33). For example, the iron oxide nanoparticle has become a research focus; this may inhibit neoplasm growth by inducing pro-inflammatory macrophage polarization (34).

A recent study has indicated that the surgical staging of cervical lymph nodes will not be replaced even by advanced imaging modalities in the next few years (35). The performance of Doppler, computed tomography, magnetic resonance imaging or positron emission tomography in the nodal staging of HNSCC is not perfect in the clinical setting. The present study revealed that SF had marked clinical importance in predicting metastasis. It is an inexpensive, routinely available 
A
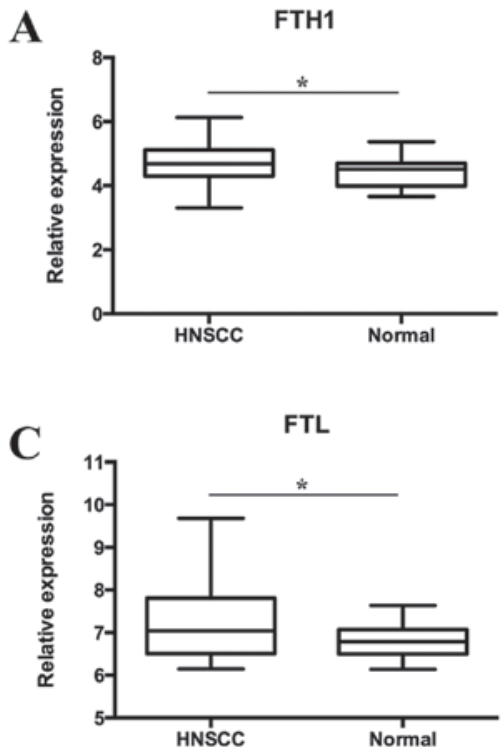

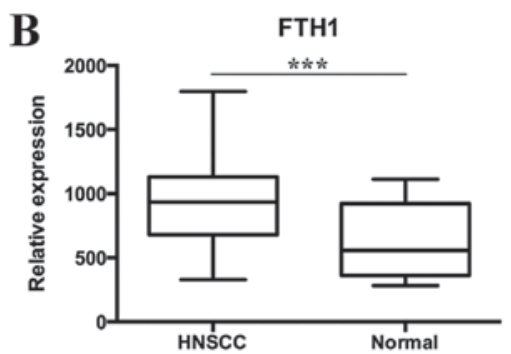

D

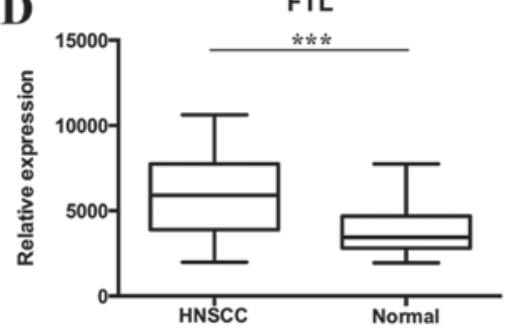

E Survival proportions: Survival of FTH1

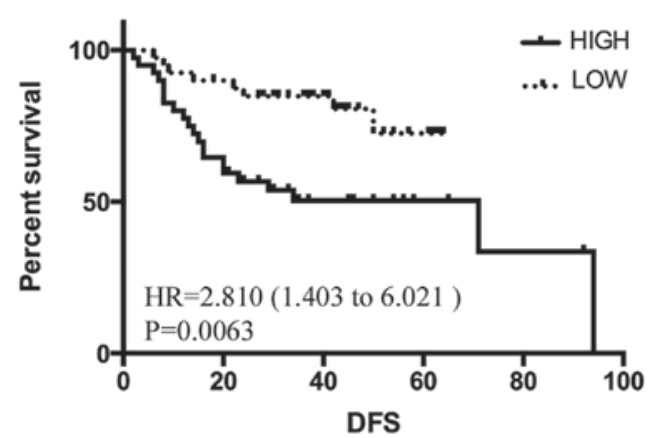

Figure 5. Analysis of the data from the GEO dataset. The data from GSE33205 demonstrated that (A) the expression level of FTH (Gene ID: 2384396) was higher in HNSCC tissues compared with normal tissues ( $\mathrm{P}=0.0397)$. The data from GSE6631 illustrated that (B) the expression level of FTH (Gene ID: 31697_s_at) was higher in HNSCC tissues compared with normal tissues ( $\mathrm{P}=0.0010)$. (C) The expression level of FTL (Gene ID: 3838094) was higher in HNSCC tissues compared with normal tissues ( $\mathrm{P}=0.0202$ ). (D) The expression level of FTL (Gene ID: 35083 at) was higher in HNSCC tissues compared with normal tissues $(\mathrm{P}=0.0010)$. (E) The data from GSE27020 demonstrated that a high expression level of FTH was associated with a shorter DFS time in patients with HNSCC [Gene ID: 200748 s_at; HR=2.810 (1.403-.021), $\mathrm{P}=0.0063]$. ${ }^{*} \mathrm{P}<0.05,{ }^{* * *} \mathrm{P}<0.001$. GEO, Gene Expression Omnibus; FTH, ferritin H subunit; FTL, ferritin L subunit; HNSCC, head and neck squamous cell carcinoma; DFS, disease free survival; HR, hazard ratio.

marker that may serve as a valuable tool and may improve predictive accuracy for lymph node metastasis of HNSCC.

In conclusion, ferritin may be used a biomarker for the early diagnosis of other malignancies, yet it may not be applicable for HNSCC. However, the association of ferritin with HNSCC may help identify patients at high risk of metastasis. Ferritin may be a novel potential biomarker for detecting cervical node metastasis in HNSCC.

\section{Acknowledgements}

Not applicable.

\section{Funding}

This study was funded by the National Natural Science Foundation of China (grant nos. 81372880 and 81172569), the Natural Science Foundation of Zhejiang Province (grant no. LY17H160065) and the Research Foundation of Zhejiang Provincial Administration of Traditional Chinese Medicine (grant no. 2016ZB018).

\section{Availability of data and materials}

The datasets used and/or analyzed during the current study are available from the corresponding author on reasonable request.

\section{Authors' contributions}

ZH,LW, CC and ZT designed the study and prepared the manuscript. LW, YH, FL, AZ and YX collected clinical samples and analyzed data. ZH, LW, FL, FW and BX conducted the experiments. AZ, YX, FW and BX critically reviewed the manuscript and supervised the study. $\mathrm{ZH}$ and LW wrote the manuscript. All authors read and approved the final manuscript.

\section{Ethics approval and consent to participate}

The present study was approved by the ethics committee of Renmin Hospital of Wuhan University. All specimens were collected from patients who provided written informed 
consent, in accordance with the principles of the Declaration of Helsinki and Good Clinical Practice Guidelines.

\section{Patient consent for publication}

Not applicable.

\section{Competing interests}

The authors declare that they have no competing interests.

\section{References}

1. Busch C, Becker B, Kriegs M, Gatzemeier F, Krüger K, Möckelmann N, Fritz G, Petersen C, Knecht R, Rothkamm K, et al: Similar cisplatin sensitivity of HPV-positive and -negative HNSCC cell lines. Oncotarget 7: 35832-35842, 2016.

2. Suarez C, Rodrigo JP, Robbins KT, Paleri V, Silver CE, Rinaldo A, Medina JE, Hamoir M, Sanabria A, Mondin V, et al: Superselective neck dissection: Rationale, indications, and results. Eur Arch Oto-Rhino-L 270: 2815-2821, 2013.

3. Vigneswaran N and Williams MD: Epidemiologic trends in head and neck cancer and aids in diagnosis. Oral Maxillofac Surg Clin North Am 26: 123-141, 2014.

4. Argiris A, Karamouzis MV, Raben D and Ferris RL: Head and neck cancer. Lancet 371: 1695-1709, 2008.

5. Trivedi S, Mattos J, Gooding W, Godfrey TE and Ferris RL: Correlation of tumor marker expression with nodal disease burden in metastatic head and neck cancer. Otolaryngol Head Neck Surg 149: 261-268, 2013.

6. SV T and FM T: Iron and cancer: More ore to be mined. Nat Rev Cancer 13: 342-355, 2013.

7. Lenarduzzi M, Hui $A B$, Yue S, Ito E, Shi W, Williams J, Bruce J, Sakemura-Nakatsugawa N, Xu W, Schimmer A, et al: Hemochromatosis enhances tumor progression via upregulation of intracellular iron in head and neck cancer. PLoS One 8: e74075, 2013.

8. Ahn SH, Lee S, Kim H, Lee SH, Kim BJ and Koh JM: Higher serum ferritin level and lower femur neck strength in women at the stage of bone loss ( $\geq 45$ years of age): The fourth Korea national health and nutrition examination survey (Knhanes IV). Endocr Res 41: 334-342, 2016.

9. Shi HB, Li XD, Jiang JT, Zhao WQ, Ji M and Wu CP: Serum ferritin is elevated in advanced non-small cell lung cancer patients and is associated with efficacy of platinum-based chemotherapy. J Cancer Res Ther 10: 681-685, 2014.

10. Zhao Y, Wang M Cui C, Zhang L, Liao F, Li H and Wu X: Significance of combined tests of serum golgi glycoprotein 73 and other biomarkers in diagnosis of small primary hepatocellular carcinoma. Cancer Biomark 15: 677-683, 2015.

11. Arenas-Salinas M, Townsend PD, Brito C, Marquez V, Marabolli V, Gonzalez-Nilo F, Matias C, Watt RK, López-Castro JD, Domínguez-Vera J, et al: The crystal structure of ferritin from chlorobium tepidum reveals a new conformation of the 4-fold channel for this protein family. Biochimie 106: 39-47, 2014.

12. Memon S, Gao RC, Jiang RZ, Si WN and Zhang XH: Deciphering the evolution of ferritin gene family in various living organisms. Pak J Agr Sci 52: 1055-1063, 2015.

13. Timoshnikov VA, Kobzeva TV, Polyakov NE and Kontoghiorghes GJ: Inhibition of $\mathrm{Fe}(2+)$ - and $\mathrm{Fe}(3+)$-induced hydroxyl radical production by the iron-chelating drug deferiprone. Free Radic Biol Med 78: 118-122, 2015.

14. Ma X, Wang X, Gao X, Wang L, Lu Y, Gao P, Deng W, Yu P, Ma J, Guo J, et al: Identification of five human novel genes associated with cell proliferation by cell-based screening from an expressed cDNA ORF library. Life Sci 81: 1141-1151, 2007.

15. Han LL, Wang YX, Li J, Zhang XL, Bian C, Wang H, Du S and Suo LN: Gender differences in associations of serum ferritin and diabetes, metabolic syndrome, and obesity in the China health and nutrition survey. Mol Nutr Food Res 58: 2189-2195, 2014.

16. Kato I, Dnistrian AM Schwartz M, Toniolo P, Koenig K, Shore RE, Zeleniuch-Jacquotte A, Akhmedkhanov A and Riboli E: Risk of iron overload among middle-aged women. Int J Vitam Nutr Res 70: 119-125, 2000.

17. Rychtarcikova Z, Lettlova S Tomkova V, Korenkova V, Langerova L, Simonova E, Zjablovskaja P, Alberich-Jorda M, Neuzil J and Truksa J: Tumor-initiating cells of breast and prostate origin show alterations in the expression of genes related to iron metabolism. Oncotarget 8: 6376-6398, 2017.
18. Pizzamiglio S, Bortoli MD, Taverna E, Signore M, Veneroni S, Cho WC, Orlandi R, Verderio P and Bongarzone I: Expression of iron-related proteins differentiate non-cancerous and dancerous breast tumors. Int J Mol Sci 18: E410, 2017.

19. Wang X, Peng A, Zeng J, Liu X, Wang B, Fang X, Wang F, Ren G, and Min J: Serum ferritin in combination with prostate-specific antigen improves predictive accuracy for prostate cancer. Oncotarget 8: 17862-17872, 2017.

20. Daher R, Manceau H and Karim Z: Iron metabolism and the role of the iron-regulating hormone hepcidin in health and disease. Presse Med 46: E272-E278, 2017.

21. Jablonska E, Socha K Reszka E, Wieczorek E, Skokowski J, Kalinowski L, Fendler W, Seroczynska B, Wozniak M, Borawska $\mathrm{MH}$, et al: Cadmium, arsenic, selenium and iron-Implications for tumor progression in breast cancer. Environ Toxicol Pharmacol 53: 151-157, 2017.

22. Jeong SM, Hwang S and Seong RH: Transferrin receptor regulates pancreatic cancer growth by modulating mitochondrial respiration and ROS generation. Biochem Biophys Res Commun 471: 373-379, 2016

23. Jeong SM, Lee J, Finley LW, Schmidt PJ, Fleming MD and Haigis MC: SIRT3 regulates cellular iron metabolism and cancer growth by repressing iron regulatory protein 1 . Oncogene 34 : 2115-2124, 2015.

24. Guo W, Zhang S, Chen Y, Zhang D, Yuan L, Cong H and Liu S: An important role of the hepcidin-ferroportin signaling in affecting tumor growth and metastasis. Acta Bioch Bioph Sin (Shanghai) 47: 703-715, 2015.

25. Chen Y, Zhang Z, Yang K, Du J, Xu Y and Liu S: Myeloid zinc-finger 1 (MZF-1) suppresses prostate tumor growth through enforcing ferroportin-conducted iron egress. Oncogene 34: 3839-3847, 2015.

26. Kell DB and Pretorius E: Serum ferritin is an important inflammatory disease marker, as it is mainly a leakage product from damaged cells. Metallomics 6: 748-773, 2014

27. Gartner A, Berger J, Bour A, El Ati J, Traissac P,Landais E, El Kabbaj S and Delpeuch F: Assessment of iron deficiency in the context of the obesity epidemic: Importance of correcting serum ferritin concentrations for inflammation. Am J Clin Nutr 98: 821-826, 2013.

28. Coffman LG, Parsonage D, D'Agostino R, Torti FM and Torti SV: Regulatory effects of ferritin on angiogenesis. Proc Natl Acad Sci USA 106: 570-575, 2009.

29. Liu NQ, De Marchi T, Timmermans AM, Beekhof R, Trapman-Jansen AM, Foekens R, Look MP, van Deurzen CH, Span PN, Sweep FC, et al: Ferritin heavy chain in triple negative breast cancer: A favorable prognostic marker that relates to a cluster of differentiation 8 positive $\left(\mathrm{CD}^{8+}\right)$ effector T-cell response. Mol Cell Proteomics 13: 1814-1827, 2014.

30. Rosager AM, Sorensen MD, Dahlrot RH, Hansen S, Schonberg DL, Rich JN, Lathia JD and Kristensen BW: Transferrin receptor-1 and ferritin heavy and light chains in astrocytic brain tumors: Expression and prognostic value. PLoS One 12: e0182954, 2017.

31. Zhukova OS, Smirnova ZS, Chikileva IO and Kiselevskii MV: Antiproliferative activity of a new nitrosyl iron complex with cysteamine in human tumor cells in vitro. Bull Exp Biol Med 162: 583-588, 2017.

32. Mahajan UM, Teller S, Sendler M, Palankar R, van den Brandt C, Schwaiger T, Kühn JP, Ribback S, Glöckl G, Evert M, et al: Tumour-specific delivery of siRNA-coupled superparamagnetic iron oxide nanoparticles, targeted against PLK1, stops progression of pancreatic cancer. Gut 65: 1838-1849, 2016.

33. Kir D, Saluja M, Modi S, Venkatachalam A, Schnettler E, Roy S and Ramakrishnan S: Cell-permeable iron inhibits vascular endothelial growth factor receptor-2 signaling and tumor angiogenesis. Oncotarget 7: 65348-65363, 2016.

34. Zanganeh S, Hutter G, Spitler R, Lenkov O, Mahmoudi M, Shaw A, Pajarinen JS, Nejadnik H, Goodman S, Moseley M, et al: Iron oxide nanoparticles inhibit tumour growth by inducing pro-inflammatory macrophage polarization in tumour tissues. Nat Nanotechnol 11: 986-994, 2016.

35. Heusch P, Sproll C, Buchbender C, Rieser E, Terjung J, Antke C, Boeck I, Macht S, Scherer A, Antoch G, et al: Diagnostic accuracy of ultrasound, ${ }^{18} \mathrm{~F}-\mathrm{FDG}-\mathrm{PET} / \mathrm{CT}$, and fused ${ }^{18} \mathrm{~F}-\mathrm{FDG}-\mathrm{PET}-\mathrm{MR}$ images with DWI for the detection of cervical lymph node metastases of HNSCC. Clin Oral Investig 18: 969-978, 2014. 